Sessions d'étude - Société canadienne d'histoire de l'Église catholique

\title{
Les collections religieuses du Musée Historique de Vaudreuil
}

\section{Andrée Boileau-De Serres}

Volume 45, 1978

URI : https://id.erudit.org/iderudit/1007124ar

DOI : https://doi.org/10.7202/1007124ar

Aller au sommaire du numéro

Éditeur(s)

Les Éditions Historia Ecclesiæ Catholicæ Canadensis Inc.

ISSN

0318-6172 (imprimé)

1927-7067 (numérique)

Découvrir la revue

Citer cet article

Boileau-De Serres, A. (1978). Les collections religieuses du Musée Historique de Vaudreuil. Sessions d'étude - Société canadienne d'histoire de l'Église catholique, 45, 57-70. https://doi.org/10.7202/1007124ar

Tous droits réservés @ Les Éditions Historia Ecclesiæ Catholicæ Canadensis Inc., 1978
Ce document est protégé par la loi sur le droit d'auteur. L'utilisation des services d’Érudit (y compris la reproduction) est assujettie à sa politique d'utilisation que vous pouvez consulter en ligne.

https://apropos.erudit.org/fr/usagers/politique-dutilisation/ 
s.C.H.E.C., Sessions d'études, 45 (1978, pp. 57-70)

\section{Les collections religieuses du Musée Historique de Vaudreuil}

(Présentation sous forme de diapositives)

\section{Musée Historique de Vaudreuil}

Le Musée a été fondé en 1953 à l'île Perrot. En 1959, la commission scolaire de Vaudreuil cède aux administrateurs du Musée l'ancienne école Saint-Michel de Vaudreuil construite de 1857 à 1859 . La Ville de Vaudreuil cède de son côté le terrain attenant.

Cet édifice a été restauré et déclaré monument historique en 1961 et c'est en 1965 qu'est ouvert officiellement le Musée Historique de Vaudreuil.

Le Musée de Vaudreuil est un Musée d'art et de tradition populaire et la majeure partie de ses collections est constituée d'instruments rattachés à la vie courante du paysan. 


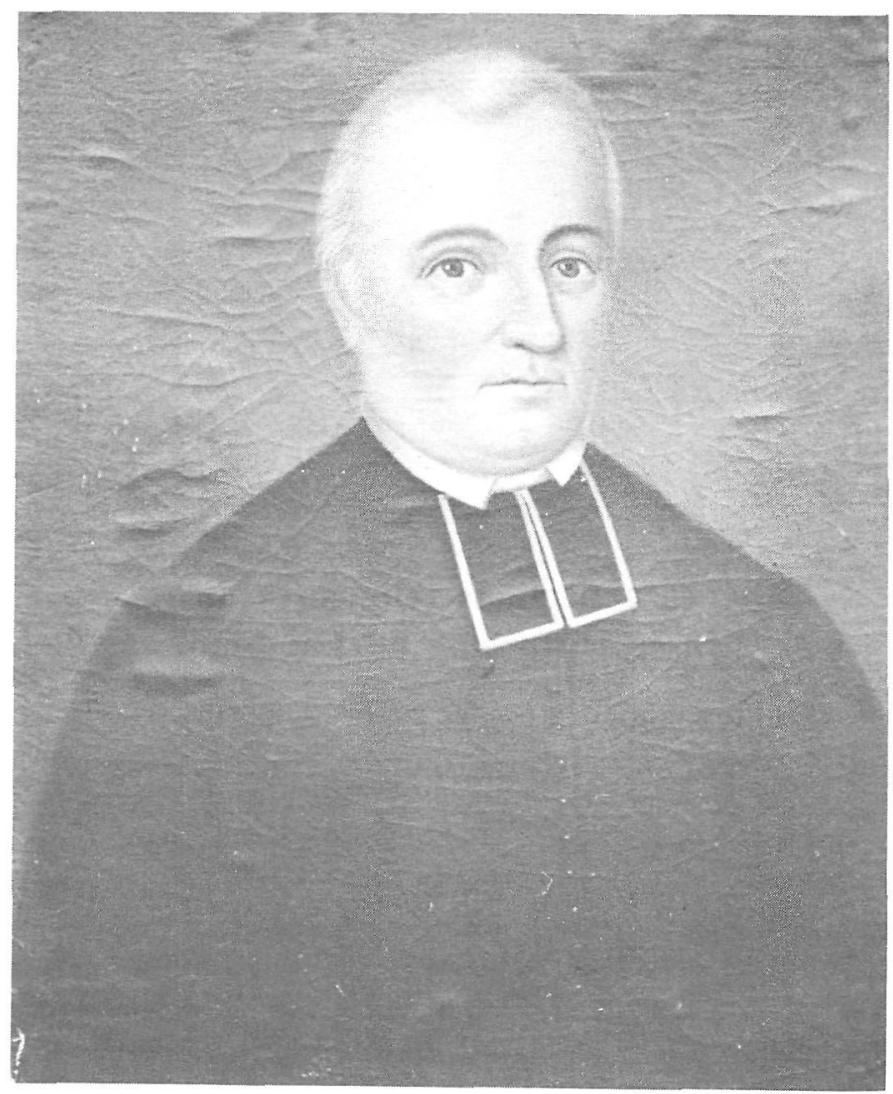

\section{Portrait du Curé Paul-Loup Archambault}

Portrait du Curé Paul-Loup Archambault, signé Collin, non daté (mais entre 1832-1869), huile sur toile, $57 \mathrm{~cm} \times 47$ $\mathrm{cm}$.

La fabrique de Vaudreuil, comprenant le rôle de conservation du Musée, a jugé bon d'y déposer quelques-unes de ses pièces dont ce portrait.

Paul-Loup Archambault fut le quatrième curé de la paroisse Saint-Michel de Vaudreuil de 1816 à 1858. Il a d'ailleurs été inhumé à Vaudreuil le 24 février 1858. Le Curé Archambault a aidé Marie-Esther Sureau-Blondin à la fondation des Sours de Sainte-Anne en 1850. 


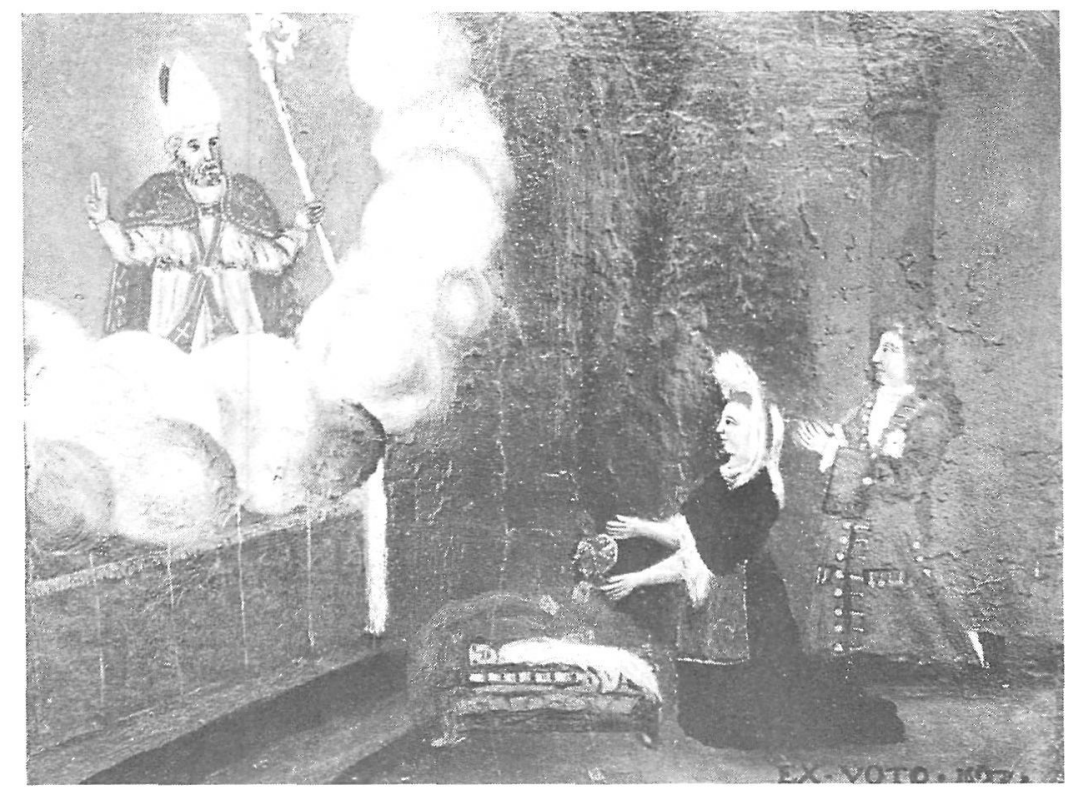

\section{Ex-voto de l'enfant malade}

Ex-voto de l'enfant malade, Peintre anonyme, 1697, huile sur bois, $30,5 \mathrm{~cm} \times 39,5 \mathrm{~cm}$.

Cet ex-voto de 1697 représente un couple, d'une famille bourgeoise d'après leur costume, implorant un saint pour la guérison de leur enfant malade. Il est intéressant de noter que le peintre a fait une double représentation de l'enfant avant et après sa guérison.

Les ex-voto sont des tableaux commémorant des miracles ou des guérisons. On les accrochait bien en vue dans les églises comme preuve tangible des pouvoirs miraculeux du saint imploré. La palette crue et l'ignorance du dessin et des lois de la perspective caractérisent tous les ex-voto à travers le monde. Ils sont sans aucun doute l'œuvre de quelques artisans locaux sans formation académique. Cette simplicité facilite d'autant plus la lecture de ces images dénuées de tout détail inutile. Tout se concentre autour de l'histoire racontée. 


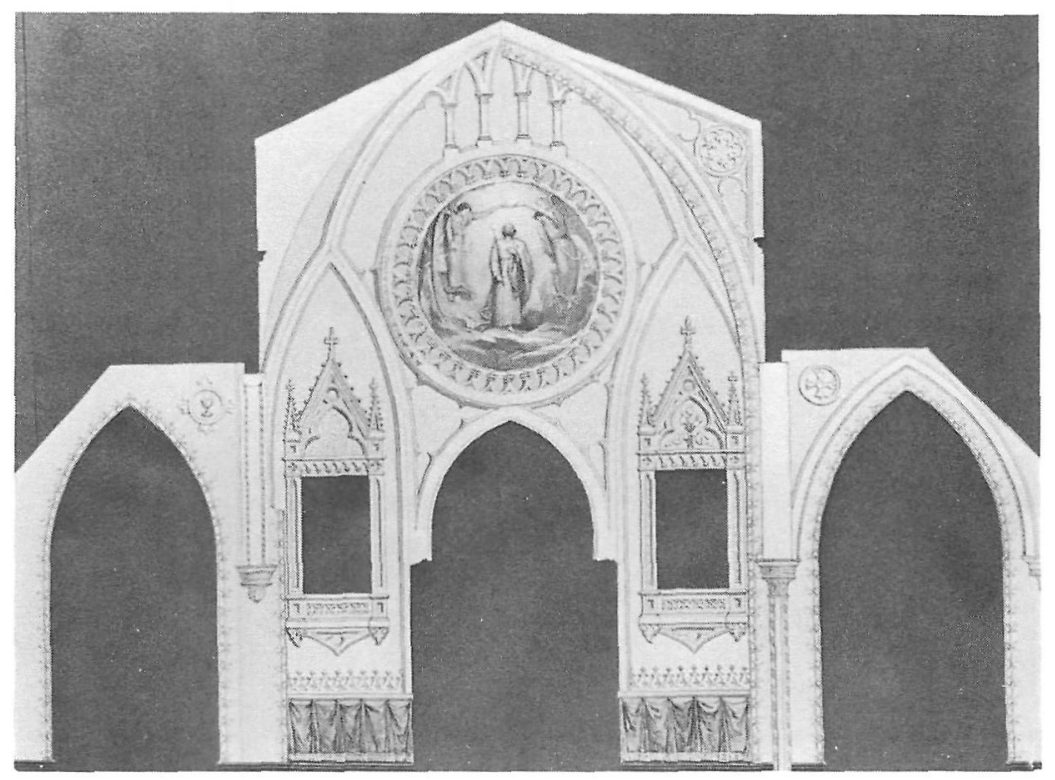

\section{Projet d'église}

Projet d'église, no. 36 par Ozias Leduc, non daté, aquarelle sur papier, $45,4 \mathrm{~cm} \times 60,5 \mathrm{~cm}$.

Ozias Leduc est né à St-Hilaire en 1864. Il meurt en 1955 à St-Hyacinthe. Il travaille un temps à la décoration d'églises avec Luigi Capelo, peintre à la mode de la région de Montréal. Parmi ses œuvres de décorations importantes, signalons la décoration intérieure de l'archevêché de Sherbrooke et le plafond du nouveau baptistère de l'église Notre-Dame de Montréal.

Ce projet de décoration d'église fait probablement partie d'une série puisqu'il comporte une inscription au dos: "Projet de décoration $20 \times 28$ no. $36 »$. Il y a de fortes chances que ce projet soit dédié à St-Joseph, puisque la rosace principale représente le couronnement, par deux anges, de St-Joseph tenant dans ses mains une branche de lys. 


\section{Maquette de l'église Notre-Dame}

La première église Notre-Dame fut construite entre 1672 et 1783, elle mesurait 144 pieds dans sa longueur et 94 pieds dans sa largeur. Elle était en pierres des champs et très massive. Elle fut démolie en 1830 et la tour subit le même sort en 1843.

Nous ignorons le nom de l'auteur de cette maquette ainsi que sa date de fabrication.

6. Maquette de l'église

St-Pierre Apôtre

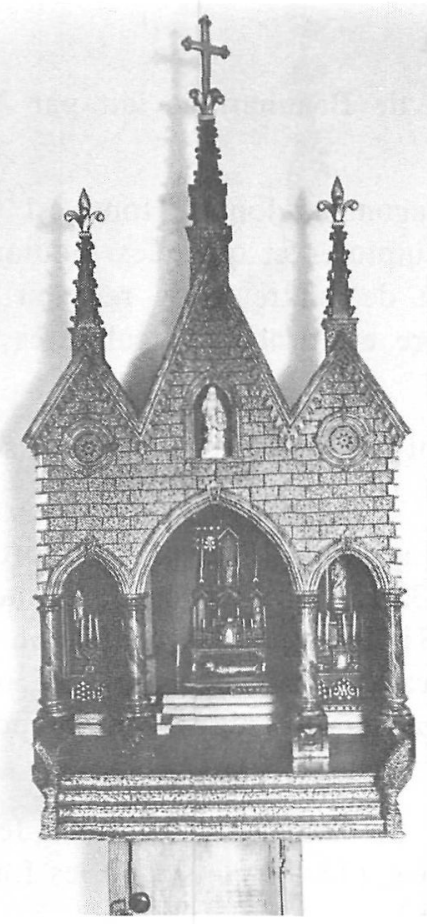

Maquette de l'église StPierre Apôtre faite par Henri Gauthier (18511911) à la fin du XIXe siècle en pin polychrome.

Henri Gauthier est un peintre-décorateur qui a vécu à Vaudreuil à la fin du siècle dernier. Cette maquette d'une église de Montréal fut probablement un projet de décoration qui n'a pas eu de suite car les seuls éléments communs de l'église avec la maquette sont les flèches surmontées de croix décorées de motifs végétaux stylisés. 


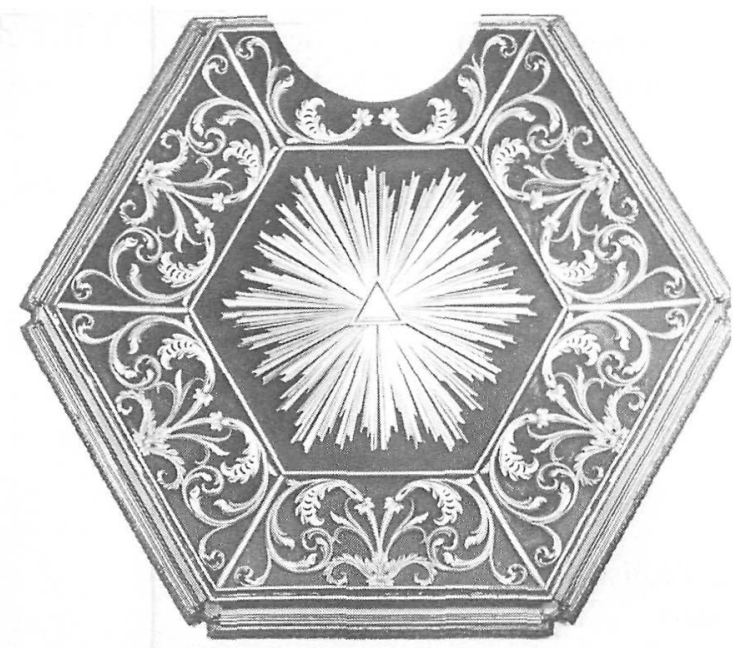

7. Abat-voix et croix de l'abat-voix

Abat-voix de l'église St-Clément de Beauharnois fait par Nicolas Manny vers 1850.

Cet abat-voix est de forme hexagonale, dont le fond est recouvert de feutre rouge et les sculptures et quelques moulures recouvertes d'une mince couche de plâtre pour recevoir la feuille d'or. La bordure extérieure est ornée de sculptures en forme de feuilles d'acanthe.

Un abat-voix est le couronnement d'une chaire à prêcher, servant à renvoyer le son de la voix vers le sol.

Nicolas Manny est né à Saint-Luc vers 1812 et est mort à Beauharnois le 9 décembre 1883. La fabrique de St-Clément a payé à Manny la somme de 131 livres pour la confection de la chaire. Enchantés par ce travail les citoyens ont voté une somme de quatre cents louis qui sera versée à Manny pour la décoration de l'autel.

Il décorera entre autres l'église de Soulanges, de Saint-Jean (1850), de Saint-Henri à Montréal (1848) ainsi que les fonts baptismaux de Boucherville (1882). 


\section{Rosace}

Cette rosace en pin provient de l'église de St-Tite des Caps et a été sculptée par André Paquette en 1810.

André Paquette fut l'élève de Baillargé.

\section{Bon Pasteur}

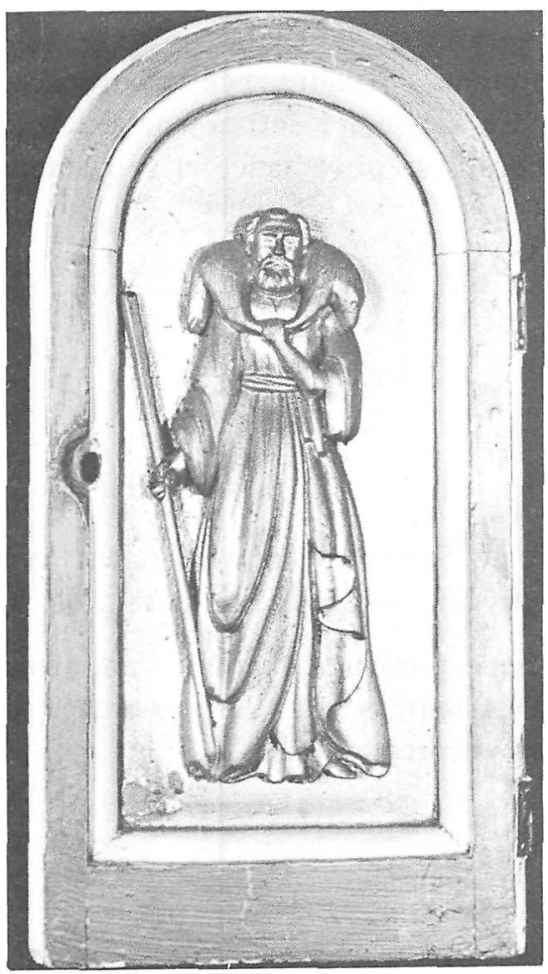

Porte de tabernacle «Bon pasteur» provenant de l'église de Ste-Marthe, non signée, XIXe siècle, bois doré et peint en blanc.

Le «Bon pasteur» de l'église de Sainte-Marthe représente un des sujets les plus naïfs de l'art québécois. Il est dû à un artiste anonyme. 


\section{Ostensoir}

Ostensoir en bois, orné de pierres décoratives, non signé, non daté.

Comme pour beaucoup d'objets de nos collections nous ignorons la provenance et l'auteur de cette pièce.

\section{Reliquaire}

Reliquaire en pin aux décorations en plâtre, peint rouge et or et doublé en velours vert. Ce reliquaire sert à enchasser les reliques. Encore là nous ignorons sa provenance et son auteur.

\section{St-François de Sales}

Sculpture en pin représentant Saint-François de Sales, évêque de Genève, fondateur des Visitandines. Hauteur: 1,57 m.

Le Musée de Vaudreuil possède plusieurs sculptures anonymes de l'art québécois religieux. Malheureusement les recherches sur leur provenance ne sont pas encore terminées.

\section{St-Ignace de Loyola}

Sculpture en pin représentant Saint-Ignace de Loyola, fondateur des Jésuites. Sculpteur anonyme. Hauteur: 1,64 m. 


\section{Sainte-Philomène}

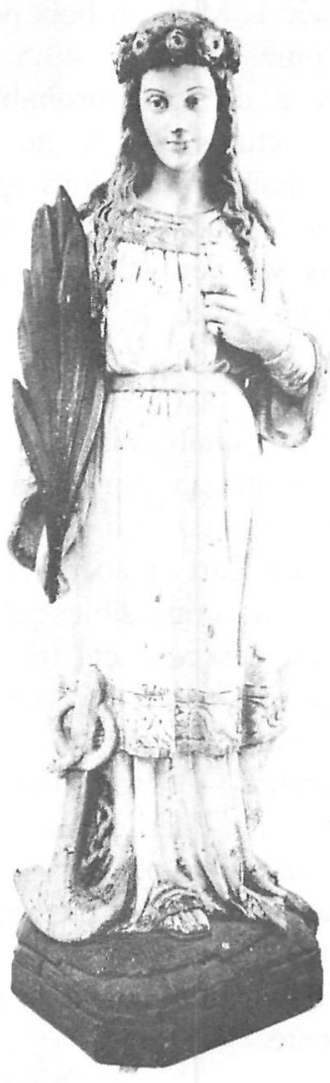

Sculpture de Sainte Philomène en pin polychrome, non signée, attribuée à Louis-Amable Quévillon. Hauteur: 1,52 m. Début du XIXe siècle.

Louis-Amable Quévillon est né au Sault-aux-Récollets en 1749. Son atelier est établi aux Écores à SaintVincent-de-Paul de l'Île Jésus. Il se rend responsable de la décoration d'une grande quantité d'édifices religieux de la région de Montréal. Son style se rattache à celui de Liebert qui est près du style de Louis XV. En 1803, la fabrique de Vaudreuil lui commande les sculptures des boiseries du sanctuaire. Il est mort en 1823.

L'histoire de Sainte Philomène commence en 1802, alors que des archéologues découvrent dans les catacombes de Rome un tombeau fragmenté. La reconstitution qu'ils en font aboutit à la découverte d'une femme portant comme attributs une ancre, une flèche, une palme et un fouet avec l'épitaphe «Filumena. Pax tecum». Déclarée martyre et sainte par l'Église, sa fête est portée au calendrier le 11 août. Récemment, les archéologues ont constaté que la reconstitution faite en 1802 était fausse. Son culte est suspendu en 1961 par la congrégation des Rites. Donc Sainte Philomène est une création des archéologues et n'a en fait jamais existé. Il n'en reste pas moins de nombreuses représentations dont celle-ci qui porte comme attributs l'ancre et la palme. 


\section{Saint-Joseph}

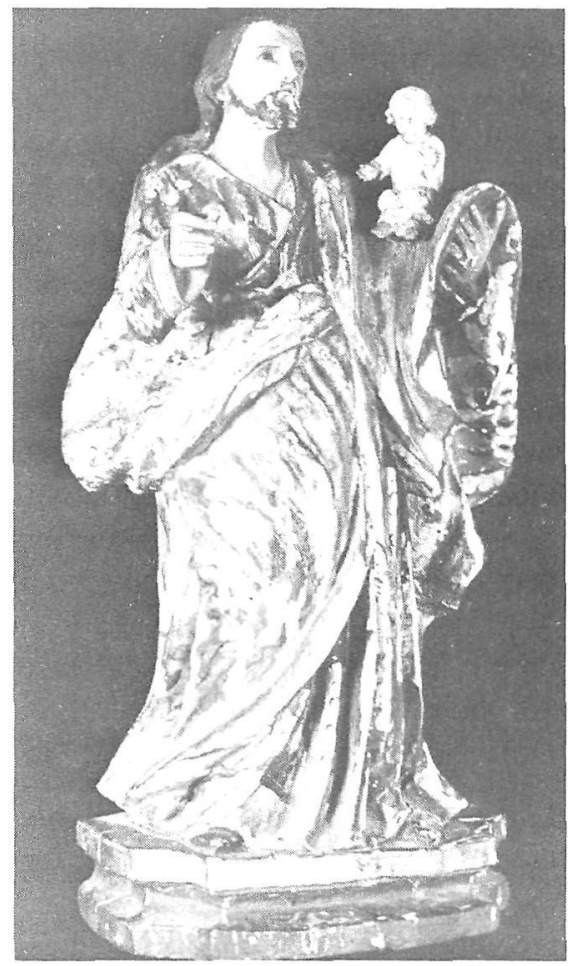

Sculpture de Saint-Joseph, signée L M A en bois polychrome. Hauteur: 43,5 cm. Il y a de forte probabilité que cette sculpture ne soit pas québécoise. Nous ignorons d'où elle provient, mais ses yeux de verre, sa polychromie, le traitement des vêtements ressemblent peu à la façon de faire des artistes québécois. On est ici beaucoup plus près de l'art colonial sud-américain que de l'art québécois. Il n'est pas impossible qu'il y ait eu des contacts très tôt entre le Québec et l'Amérique du Sud et que cette sculpture se soit trouvée dans une des églises du Québec. Il se peut aussi que cette sculpture ait été acquise en Amérique du Sud ou en Espagne à une date très récente. 


\section{Bénitiers}

De haut en bas, de gauche à droite.

- Bénitier en porcelaine, représentant un Christ sur la croix, au pied de laquelle se traîne une brebis.

- Bénitier d'applique en céramique émaillée, orné d'un cœur transpercé d'une croix.

- Bénitier d'applique en terre cuite, avec un médaillon orné de 8 têtes d'anges, entourant un Christ noir.

- Bénitier d'applique en céramique, peint bleu et jaune composé d'une plaque représentant un Christ en croix entouré d'un motif végétal et d'un bassin à la base.

- Bénitier en for blanc, composé d'un bassin décoré d'un motif floral surmonté d'une croix.

- Bénitier d'applique en céramique émaillée polychrome, composé d'un bassin surmonté d'une plaque ornée d'un ostensoir.

- Bénitier d'applique en fer plaqué argent, orné d'une plaque découpée représentant le Christ sur la croix.

- Bénitier d'applique en bronze poli, composé d'un bassin mouluré surmonté d'une plaque ajourée décorée de volutes et de spirales.

- Bénitier d'applique en céramique émaillée polychrome. Le dais s'ouvre sur un Christ en croix, sur fond à motif floral.

- Bénitier en faïence composé d'un bassin bombé surmonté d'un ange agenouillé.

- Bénitier d'applique en céramique émaillée polychrome composé d'un bassin surmonté d'un dais s'ouvrant sur un Christ en croix, en retrait.

- Bénitier d'applique dont le bassin est fait d'un tridacne blanc, cloué à une planche de bois et surmonté d'une plaque de plâtre moulurée, dans laquelle est insérée une croix sur laquelle est cloué un Christ, recouvert d'une vitre bombée décorée autour d'un ruban de fil doré.

- Bénitier d'applique en céramique émaillée blanc, bleu et jaune, composé d'un bassin surmonté d'une plaque en forme de croix sur laquelle est peint un Christ en croix. 


\section{Bénitiers}

De haut en bas, de gauche à droite.

- Bénitier d'applique composé d'un bassin en marbre orné de rainures, surmonté d'une plaque ajourée, en onyx, représentant une croix entourée de feuilles se terminant à une extrémité par des volutes.

- Bénitier d'applique dont le bassin en forme de coquillage et la plaque ornée de volutes ajourées peinte d'un motif floral, ont été sculptés dans une seule pièce d'onyx.

- Bénitier d'applique en laiton dont le bassin mouluré est soudé à une plaque ornée d'une tête d'ange et de volutes ajourées entourant un médaillon portant une inscription et représentant un pape.

- Bénitier d'applique en verre transparent. Le bassin est surmonté d'une plaque ajourée, décorée de feuilles et de torsades.

- Bénitier d'applique en bronze, le bassin est surmonté d'une plaque ajourée représentant le Christ sur la croix, entouré des saintes femmes.

- Bénitier d'applique en faïence. Une Croix aux extrémités trèflées est peinte sur une plaque.

- Bénitier d'applique en étain orné d'un Christ en croix entouré des saintes femmes.

- Bénitier d'applique en verre blanc, avec plaque peinte d'une croix dorée.

- Bénitier d'applique en plâtre émaillé représentant Saint-Michel et le dragon.

- Bénitier d'applique en faïence dont le bassin est décoré à une extrémité d'une tête d'ange et à l'autre de festons.

- Bénitier d'applique en verre blanc dont le bassin se prolonge en une plaque découpée représentant Ste-Anne. La plaque se termine par une croix entourée de roses. 


\section{Chapelet}

Chapelet en bois dont les grains de forme ovale sont reliés entre eux par les mailles de broches. Une boule sculptée de cercles s'enlaçant autour d'un trou et placé entre deux grains en forme de cloche, sépare chaque dizaine et précède un coeur auquel sont reliées les deux extrémités des cinq dizaines. Inscription : "Cour Immaculé de Marie, protégez-nous 》.Le crucifix dont les extrémités sont chantournées est tiré d'une seule pièce et muni d'un Christ en laiton cloué. Inscription : "Souvenir de Ste-Anne de Beaupré ».

Longueur : $1,40 \mathrm{~m}$.

\section{Vierge au blé}

Sculpture de la Vierge au blé en pin exécutée par Médard Bourgault.

Médard Bourgault est né le 9 juin 1890 et est mort à St-Jean Port-Joli en 1960 à l'âge de 70 ans. Après avoir commencé à sculpter des personnages qui l'entouraient, il en vient à l'art religieux, témoignage de sa ferveur religieuse. Ses pièces ornent maintes églises canadiennes et même quelques sanctuaires à l'étranger. Ses Christ en croix et ses stations de chemin de la croix lui ont apporté la majeure partie de sa célébrité. Il n'abandonnera toutefois jamais l'art profane et initiera ses frères André et Jean-Julien au métier.

Cete sculpture de la Vierge au blé a été achetée par un paroissien de l'île Perrot et donnée par la suite au Chanoine Lionel Groulx pour orner son autel. 


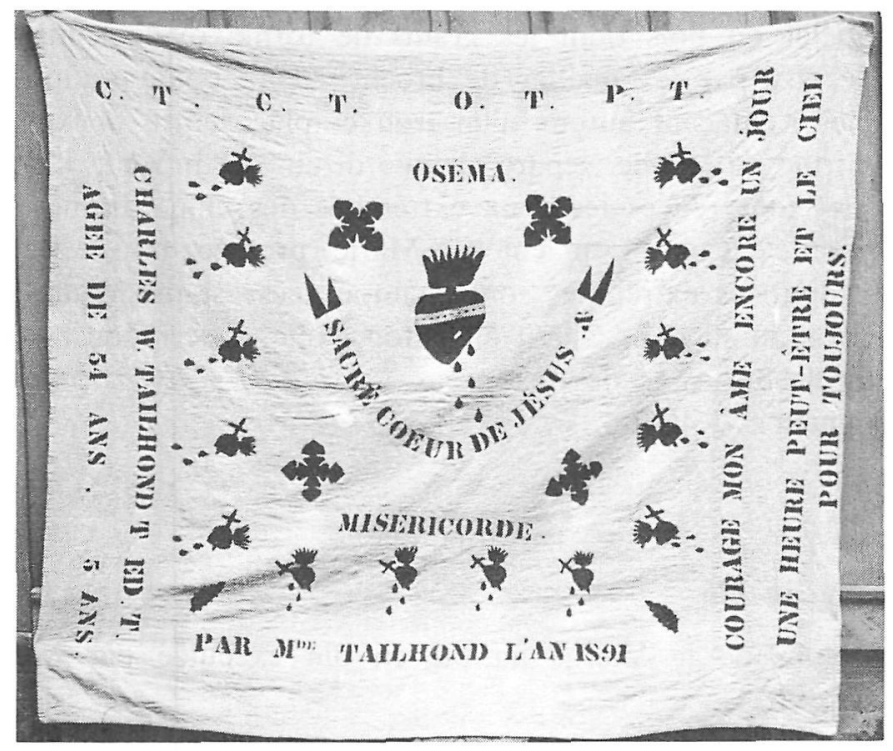

\section{Linceul}

Linceul en coton piqué blanc décoré de cœurs saignants de couleur rouge portant l'inscription sur toute sa surface :
« C. T.
C. T.
O. T.
P. T. ». En haut «OSEMA»
«Sacré-Cœur de Jésus Miséricorde» (au centre)
«par Mde Tailhond l'an 1891 ( au bas)
«Courage mon âme encore un jour

Une heure peut-être et le ciel pour toujours »

«Charles W. Tailhond T. ed T. (sur une bordure) âgée de 54 ans. 5 ans.» (sur l'autre bordure)

Le linceul est une pièce de tissu, décorée ou non, dans laquelle on enveloppait et ensevelissait les morts. En ce qui concerne ce linceul, il n'a dû servir qu'à recouvrir le mort pour son exposition. Il a été exécuté par Madame Tailhond en 1891 pour recouvrir le corps de Charles Tailhond décédé à l'âge de 54 ans.

Andrée B. De Serres, Administrateur déléguée Musée historique de Vaudreuil 